\title{
Secondary stroke prevention and primary care physicians
}

\section{Opinion}

Globally, more than 10 million strokes occur annually and there are almost 30 million people living with a history of stroke. ${ }^{1}$ In 2013, stroke was the second most common cause of death and third most common cause of disability worldwide, and this burden is increasing especially in developing countries. ${ }^{2}$ Approximately $90 \%$ of the risk of stroke is attributable to 10 modifiable risk factors, with high blood pressure implicated in almost $50 \%$ of stroke cases. ${ }^{3}$ Survivors of stroke are at a high risk of subsequent vascular events, including recurrent stroke ( $\sim 11 \%$ at 1 year and $26 \%$ at 5 years) and many have modifiable risk factors that are poorly managed. ${ }^{4,5}$ Primary and secondary stroke prevention is paramount to reducing the burden of stroke and primary care physicians have essential roles in supporting their patients to avoid stroke. In this paper we focus on the topic of secondary prevention in primary care.

Primary care physicians have an essential role in post-acute care management and secondary prevention of patients with stroke and TIA after they are discharged from hospital. In Australia, longterm management is by family doctors with some patients receiving additional specialist (e.g. neurologist, geriatrician or cardiologist) support as required. Often, standard procedures at hospitals exist to facilitate follow-up appointments with primary care physicians and the patient. For example, discharge letters and discharge care plans are provided to patients for discussion with their primary care physicians. The ideal timing of these follow-up consultations with primary care physicians is not described in the literature and opportunities for stroke prevention may be delayed if patients are unsure about when to see their local doctor. Better ways to monitor continuity of care after stroke is required so patients in the community who may be missing out on recommended care can be easily identified. Especially since secondary prevention therapies may not have started during the hospital stay.

There is evidence from the Australian Stroke Clinical Registry (www.auscr.com.au) that hospitals sometimes do not prescribe secondary prevention medications to eligible patients. In our recent research, we found that many hospitals appeared to have room for improvement compared to the benchmark for the provision of secondary prevention medications. While the best hospitals prescribed antihypertensive medications to $79 \%$ of patients, some hospitals prescribed this to less than $50 \%$ of patients. The best hospitals prescribed antithrombotic medications and lipid-lowering medications to more than $80 \%$ of patients, but again there were other hospitals with considerably lower proportions of patients prescribed. Few cases have contraindications as reasons for not prescribing since these data began to be collected in July 2016. There are considerable health benefits that would be expected from improving secondary stroke prevention to that of achievable benchmarks in Australia. ${ }^{6}$ Importantly, these benefits from improving secondary prevention practices were estimated to result in a cost saving to society. When patients are not prescribed these medications in hospital, prompt identification and treatment by primary care physicians would be required to realise these benefits.
Volume I Issue I - 2017

\author{
Joosup Kim, Dominique A Cadilhac \\ Department of Neuroscience and Mental Health, Monash \\ University, Australia
}

Correspondence: Dominique Cadilhac, Department of Neuroscience and Mental Health, Monash University, Australia, Tel +6I-3-8572-2657, Email Dominique.cadilhac@monash.edu

Received: August 01, 2017| Published: August 22, 2017

Primary care physicians also have the responsibility of supporting patient adherence to medications. Adherence to (or compliance with) a medication regimen is the extent to which patients take medications as prescribed. ${ }^{7}$ Poor adherence to medications prescribed for the prevention of stroke has been described in a study conducted in Canada. ${ }^{8}$ At 1 year after stroke, 62 to $76 \%$ of patients had $\geq 80 \%$ of days covered on a medication. Improving adherence is vital since there is evidence that better adherence to medications is associated with better outcome. ${ }^{9}$ Strategies to improve adherence to medication include management of side effects. ${ }^{10}$ The use of multidrug combinations often produces greater and timely BP reduction at lower doses while minimizing side effects. ${ }^{11}$ A combination of lifestyle interventions with pharmacotherapy may also minimise the need for pharmacotherapy and any side effects. ${ }^{12}$ Reminders, monitoring and feedback, provision of information, simplifying the course of medication and active education have been shown to improve adherence to medications. ${ }^{13-16}$ To improve long-term adherence to lifestyle changes and medications, there is some evidence for the use of extended care, skills training, social support, individualised management and self-monitoring. ${ }^{17}$

Patient education may also be important for improving adherence to medications since adherence to medications is affected by attitudes. Patients who perceived the benefits derived from medication to be low were found to be less likely to be adherent when compared to patients who believed that the benefits of their medications were outweighed by the negatives. ${ }^{19}$ In a study of patients who had suffered a stroke, those who reported poor adherence to medications more often believed that their medications were not useful. ${ }^{20}$ Greater persistence with secondary prevention medications was found to be associated with a better understanding of medications. ${ }^{21,22}$ In our research, we have shown that patients with stroke often report long-term unmet needs with secondary prevention, ${ }^{23}$ and that nurse-led education can improve knowledge of secondary prevention medications. ${ }^{24}$

Support from other clinicians such as specialists, nurses, dieticians and physiotherapists should also be considered for primary care physicians. In Australia, financial incentives exist for primary care physicians to plan, co-ordinate and review the management of patients with chronic diseases such as stroke, including provisions to make referrals to allied health services. These financial incentives were leveraged in a clinical trial of an individualised management programme. ${ }^{25}$ In this study, primary care physicians responsible for 
the management of patients in the intervention arm of the study were sent individualised management plans for their patients that were developed in consultation with independent stroke specialists. Primary care physicians were able to claim a government re-imbursement for implementing the plan. No significant differences were found between treatment arms in terms of the primary outcome of cardiovascular risk at 12 months follow-up. Further research is required to identify models of care that are effective in primary care.

\section{Conclusion}

There is an important role for primary care physicians in the secondary prevention stroke. Having effective treatment pathways from hospital to primary care will ensure continuity of care and minimise missed opportunities for secondary prevention. Interventions that support primary care physicians that are evidence-based are also required.

\section{Acknowledgements}

None.

\section{Conflict of interest}

The author declares no conflict of interest.

\section{References}

1. Barker Collo S, Bennett DA, Krishnamurthi RV, et al. Sex Differences in Stroke Incidence, Prevalence, Mortality and DALYs: Results from the Global Burden of Disease Study 2013. Neuroepidemiology 2015;45(3):203-214

2. Feigin VL, Norrving B, Mensah GA. Global Burden of Stroke Circulation Research. 2017;120:439-448.

3. O Donnell MJ, Chin SL, Rangarajan S, et al. Global and regional effects of potentially modifiable risk factors associated with acute stroke in 32 countries (INTERSTROKE): a case-control study. The Lancet 2016;388(10046):761-775.

4. Heuschmann PU, Kircher J, Nowe T, et al. Control of main risk factors after ischaemic stroke across Europe: data from the strokespecific module of the EUROASPIRE III survey. Eur J Prev Cardiol. 2014;22(10):1354-1362

5. Mohan KM, Wolfe CD, Rudd AG, et al. Risk and Cumulative Risk of Stroke Recurrence. Stroke. 2011;42(5):1489-1494.

6. Kim J, Andrew NE, Thrift AG, et al. The potential health and economic impact of improving stroke care standards for Australia. International Journal of Stroke. 2017;12(8):875-885

7. Osterberg L, Blaschke T. Adherence to Medication. $N$ Engl J Med. 2005;353(5):487-497.

8. Khan NA, Yun L, Humphries K, et al. Antihypertensive Drug Use and Adherence After Stroke: Are There Sex Differences? Stroke. 2010;41(1):1445-1449.
9. Chowdhury R, Khan H, Heydon E, et al. Adherence to cardiovascular therapy: a meta-analysis of prevalence and clinical consequences. Eur Heart J. 2013;34(38):2940-2948.

10. Ho PM, Bryson CL, Rumsfeld JS. Medication adherence: its importance in cardiovascular outcomes. Circulation. 2009;119(23):3028-3035.

11. Chobanian AV, Bakris GL, Black HR, et al. Seventh Report of the Joint National Committee on Prevention, Detection, Evaluation, and Treatment of High Blood Pressure. Hypertension. 2003;42(6):1206-1252.

12. Huupponen R, Viikari J. Statins and the risk of developing diabetes BMJ. 2013;346:f3156.

13. Kripalani S, Yao X, Haynes RB. Interventions to enhance medication adherence in chronic medical conditions: a systematic review. Arch Intern Med. 2007;167(6):540-550.

14. Haynes RB, Ackloo $\mathrm{E}$, Sahota $\mathrm{N}$, et al. Interventions for enhancing medication adherence. Cochrane Database Syst Rev. 2008;16(2):CD000011

15. Schroeder K, Fahey T, Ebrahim S. Interventions for improving adherence to treatment in patients with high blood pressure in ambulatory settings. Cochrane Database Syst Rev. 2004;(2):CD004804.

16. van Dulmen S, Sluijs E, van Dijk L, et al. Patient adherence to medical treatment: a review of reviews. BMC Health Serv Res. 2007;7:55.

17. Middleton KR, Anton SD, Perri MG. Long-Term Adherence to Health Behavior Change. Am J Lifestyle Med. 2013;7(6):395-404.

18. Chambers JA, O Carroll RE, Hamilton B, et al. Adherence to medication in stroke survivors: a qualitative comparison of low and high adherers. $\mathrm{Br}$ J Health Psychol. 2011;16(3):592-609.

19. Sjölander M, Eriksson M, Glader EL. The association between patients' beliefs about medicines and adherence to drug treatment after stroke: a cross-sectional questionnaire survey. BMJ Open. 2013;3(9):e003551.

20. Bushnell CD, Zimmer LO, Pan W, et al. Persistence With Stroke Prevention Medications 3 Months After Hospitalization. Arch Neurol. 2010;67(12):1456-1463.

21. Bushnell CD, Olson DM, Zhao X, et al. Secondary preventive medication persistence and adherence 1 year after stroke. Neurology. 2011;77(12):1182-1190.

22. Olaiya MT, Cadilhac DA, Kim J, et al. Long-term unmet needs and associated factors in stroke or TIA survivors: An observational study. Neurology. 2017;89(1):68-75.

23. Olaiya MT, Cadilhac DA, Kim J, et al. Nurse-Led Intervention to Improve Knowledge of Medications in Survivors of Stroke or Transient Ischemic Attack: A Cluster Randomized Controlled Trial. Front Neurol. 2016;7:205 p.

24. Olaiya MT, Kim J, Nelson MR, et al. Effectiveness of a shared team approach between nurses and doctors for improved risk factor management in survivors of stroke: a cluster randomized controlled trial. European Journal of Neurology. 2017;24(7):920-928. 